\title{
Neue Studiendaten zur Therapie der tumorassoziierten venösen Thromboembolie mit DOAKs
}

\author{
M. Voigtlaender ${ }^{1}$; J. Yamamura ${ }^{2}$; F. Langer ${ }^{1}$ \\ ${ }^{1}$ II. Medizinische Klinik und Poliklinik, Hubertus Wald Tumorzentrum - Universitäres Cancer Center Hamburg (UCCH), \\ Universitätsklinikum Hamburg-Eppendorf; ${ }^{2}$ Klinik und Poliklinik für Diagnostische und Interventionelle Radiologie und \\ Nuklearmedizin, Universitätsklinikum Hamburg-Eppendorf
}

\begin{abstract}
Schlüsselwörter
Direkte orale Antikoagulantien, niedermolekulares Heparin, tumorassoziierte venöse Thromboembolie, Blutungen, gastrointestinaler Tumor
\end{abstract}

\section{Zusammenfassung}

Die venöse Thromboembolie (VTE) ist eine häufige Komplikation bei Patienten mit maligner Grunderkrankung. Basierend auf einer verbesserten Wirksamkeit und Sicherheit gegenüber Vitamin K-Antagonisten (VKA) empfehlen aktuelle Leitlinien eine 3- bis 6-monatige Antikoagulation mit niedermolekularem Heparin (NMH) als Standardtherapie der tumorassoziierten VTE. Versorgungsdaten zeigen jedoch eine geringe Leitlinienadhärenz im klinischen Behandlungsalltag, was am ehesten auf die täglichen subkutanen Injektionen und die relativ hohen Therapiekosten zurückzuführen ist. Aufgrund ihres Einnahmemodus und ihrer im Vergleich zu VKA günstigen Pharmakokinetik stellen die direkten oralen Antikoagulantien (DOAKs) zwar eine vielversprechende Alternative dar; die Subgruppen der in die großen Zulassungsstudien eingeschlossenen Krebspatienten waren aber aufgrund ihrer Größe und Tumorcharakteristika nicht geeignet, die bisherigen Studienda- ten in den klinischen Alltag zu übertragen. Mit HOKUSAI VTE Cancer (Edoxaban) und SELECT-D (Rivaroxaban) stehen nun die Ergebnisse von zwei prospektiven, randomisierten Studien zur Verfügung, die die Wirksamkeit und Sicherheit von DOAKs im Vergleich zu NMH bei Patienten mit tumorassoziierter VTE untersucht haben. In beiden Studien war die DOAK-Einnahme mit weniger VTE-Rezidiven, jedoch mit einer höheren Rate an (gastrointestinalen und urothelialen) Blutungen assoziiert. Somit wird die zukünftige Therapie der tumorassoziierten VTE unter Berücksichtigung von Patientenpräferenz und Tumorcharakteristika ein hohes Maß an Selektion und Individualisierung erfordern.

\section{Keywords}

Direct oral anticoagulants, low-molecularweight heparin, cancer-associated venous thromboembolism, bleeding, gastrointestinal cancer

\section{Summary}

Venous thromboembolism (VTE) is a frequent complication in patients with malignancy. Based on an improved safety and efficacy profile compared to vitamin $\mathrm{K}$ antagonists (VKA), current guidelines recommend anticoagulation with low-molecular-weight heparin (LMWH) for 3-6 months as the preferred treatment of cancer-associated VTE. Realworld evidence indicates, however, that guideline adherence is poor in clinical practice, a finding most likely explained by the daily subcutaneous injections and relatively high treatment costs associated with LMWH therapy. Although direct oral anticoagulants (DOACs) may be an attractive alternative owing to their ease of administration and favorable pharmacokinetics compared to VKA, the rather small and insufficiently characterized subgroups of cancer patients included in the large phase-3 trials did not allow translation of study findings into daily practice. With HOKUSAI VTE Cancer (edoxaban) and SELECT-D (rivaroxaban) two large prospective, randomized trials, which have compared DOACs with LMWH for the treatment of cancer-associated VTE, are now available. Both studies showed a reduction in recurrent VTE, but an increased risk in (gastrointestinal and urothelial) bleeding. Taking into account patient preferences and tumor characteristics, future treatment of cancer-associated VTE will thus require a high degree of selection and individualization.
Korrespondenzadresse

Prof. Dr. med. Florian Langer

II. Medizinische Klinik und Poliklinik

Hubertus Wald Tumorzentrum - Universitäres Cancer

Center Hamburg (UCCH)

Universitätsklinikum Eppendorf

Martinistr. 52, D-20246 Hamburg

Tel. +49 40/7410-52453/-50664

Fax +49 40/7410-55193

E-Mail: langer@uke.de
Novel clinical trial data on the treatment of cancer-associated venous thromboembolism with DOACS

Phlebologie 2018; 47: 309-317

https://doi.org/10.12687/phleb2450-6-2018

Eingegangen: 26. Juli 2018

Angenommen: 15. August 2018

English version available at:

www.thieme.de/phlebo 


\section{Einleitung}

Die Behandlung der tumorassoziierten venösen Thromboembolie (VTE) stellt im klinischen Alltag eine große Herausforderung dar: Krebspatienten mit tiefer Venenthrombose (TVT) oder Lungenarterienembolie (LAE) haben unter der Antikoagulation nicht nur ein erhöhtes thromboembolisches Rezidiv-, sondern zugleich auch ein signifikant gesteigertes Blutungsrisiko im Vergleich zu Patienten ohne maligne Grunderkrankung (1-3).

Bei einer rezidivierten, lokal fortgeschrittenen oder metastasierten Tumorerkrankung handelt es sich meist um einen persistierenden schwerwiegenden Risikofaktor, sodass sich nach stattgehabter VTE in der Regel die Indikation zur langfristigen, zeitlich unbegrenzten Fortführung der Antikoagulation ergibt ( $>$ Abb. 1 und Ref. 4). Vor diesem Hintergrund kommt der Sicherheit der antikoagulatorischen Therapie bei Krebspatienten eine besondere Bedeutung zu.

Bei Patienten mit tumorassoziierter VTE hat sich die orale Antikoagulation mit Vitamin K-Antagonisten (VKA) aufgrund des hohen Blutungs- und VTE-Rezidivrisikos als nicht praktikabel erwiesen (1-3). Hierfür verantwortlich sind vor allem die ungünstige Pharmakokinetik mit verzögertem Wirkungseintritt und langer Abklingdauer, der indirekte, Vitamin K-abhängige Wirkmechanismus sowie die zahlreichen Nahrungsmittel- und Medikamenteninteraktionen, die eine stabile Einstellung der
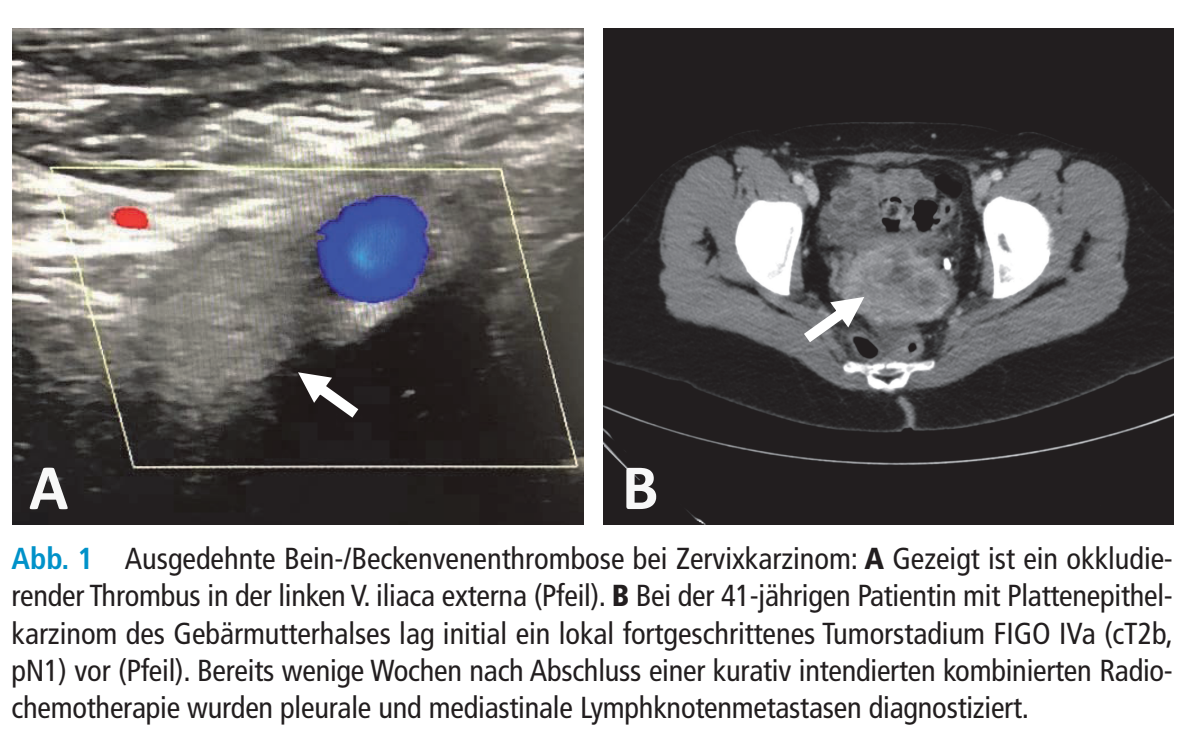

Abb. 1 Ausgedehnte Bein-/Beckenvenenthrombose bei Zervixkarzinom: A Gezeigt ist ein okkludierender Thrombus in der linken V. iliaca externa (Pfeil). B Bei der 41 -jährigen Patientin mit Plattenepithelkarzinom des Gebärmutterhalses lag initial ein lokal fortgeschrittenes Tumorstadium FIGO IVa (cT2b, pN1) vor (Pfeil). Bereits wenige Wochen nach Abschluss einer kurativ intendierten kombinierten Radiochemotherapie wurden pleurale und mediastinale Lymphknotenmetastasen diagnostiziert. international normalisierten Ratio (INR) bei Tumorpatienten besonders erschweren. Basierend auf mehreren prospektiven randomisierten Studien empfehlen nationale und internationale Leitlinien eine Antikoagulation mit niedermolekularem Heparin (NMH) über 3-6 Monate als Standardtherapie der tumorassoziieren VTE (5). Metaanalysen zeigen, dass die $\mathrm{NMH}$ - gegenüber der VKA-Therapie das VTE-Rezidivrisiko bei Tumorpatienten signifikant um 40-50 \% reduziert, ohne dabei das Risiko für schwere Blutungen zu erhöhen (6-8).

Wichtig in diesem Zusammenhang ist jedoch die Anmerkung, dass die verbesserte Wirksamkeit von NMH im Vergleich zu VKA im Wesentlichen auf den Ergebnissen der CLOT-Studie beruht, in der durch eine sechsmonatige Antikoagulation mit Dalteparin das VTE-Rezidivrisiko von $17 \%$ im VKA-Arm auf $9 \%$ im NMH-Arm gesenkt werden konnte, entsprechend einer relativen Risikoreduktion von $52 \%(\mathrm{P}=0,002)$ (9). Keine andere NMH-Studie, inklusive der bisher größten Studie CATCH (Tinzaparin vs. Warfarin) (10), hat für sich genommen eine überlegene Wirksamkeit von $\mathrm{NMH}$ gegenüber VKA gezeigt. Entsprechend unterschiedlich wird die Evidenz in den Leitlinien bewertet. Während die nordamerikanische ACCP-Leitlinie zur VTE-Therapie bei Tumorpatienten NMH anstelle von VKA mit einer Grad 2B-Empfehlung vorschlagen (11), wird in der ITAC-CME-Leitlinie die NMH- gegenüber der VKA-Therapie mit einer Grad 1A-Empfehlung bevorzugt (12).
Versorgungsdaten zeigen, dass die Umsetzung der Leitlinienempfehlungen im klinischen Behandlungsalltag mangelhaft ist. So wird in den USA unverändert ein Großteil der onkologischen VTE-Patienten mit VKA therapiert, worunter die Therapiepersistenz im Vergleich zur parenteralen Antikoagulation mit NMH deutlich verbessert ist $(13,14)$. In einer Studie von Khorana und Mitarbeitern lag die mediane Behandlungsdauer bei 3,3 Monaten in der NMHund bei 7,9 Monaten in der VKA-Kohorte (14). Nach sechs Monaten hatten bereits $63 \%$ der NMH-Patienten die initial verordnete Therapie beendet, während dies bei nur 39 \% der VKA-Patienten der Fall war. In Deutschland zeigt sich ein ähnliches Bild. In einer Umfrage unter Hämatologen/Onkologen, Angiologen und Phlebologen gaben nur $55 \%$ der Ärzte an, in der Phase der Sekundärprophylaxe einer tumorassoziierten VTE (d. h. während der ersten 3-6 Monate) auf den Einsatz oraler Antikoagulantien zu verzichten (15). Auch wenn die erhöhten Kosten der NMH-Therapie und insbesondere in den USA erstattungsrechtliche Aspekte als Gründe für die ungenügende Leitlinienadhärenz in Frage kommen, müssen neben Spritzenangst und bekannter Heparinallergie oder heparininduzierter Thrombozytopenie (HIT) die täglichen subkutanen Injektionen mit schmerzafter Hämatombildung und negativen Effekten auf die Lebensqualität onkologischer Patienten berücksichtigt werden.

Hervorzuheben sind zudem Berichte, nach denen bei Krebspatienten mit VTE eine orale VKA-Therapie ausreichend wirksam und sicher scheint, wenn diese durch spezialisierte Einrichtungen mit dem Ziel einer bestmöglichen INR-Einstellung begleitet wird $(16,17)$.

Vor diesem Hintergrund ergab sich aus der Markteinführung der direkten oralen Antikoagulation (DOAKs) die Hoffnung, Patienten mit tumorassoziierter VTE eine sichere und wirksame Therapieoption anbieten zu können, die die Vorteile von VKA (orale Tabletteneinnahme und geringere Therapiekosten) mit denen von $\mathrm{NMH}$ (berechenbare Pharmakokinetik mit schnellem Wirkungseintritt, kurzer Abklingdauer und geringen Nahrungsmittel-/ Medikamenteninteraktionen) verbindet. Explorative Subgruppenanalysen der in die 
großen Zulassungsstudien eingeschlossenen Tumorpatienten haben gezeigt, dass DOAKs eine sinnvolle Alternative zu VKA in der Therapie der tumorassoziierten VTE darstellen könnten (18). Die Subgruppen der onkologischen Patienten, die jeweils nur etwa 5-6 \% der gesamten Studienpopulation ausmachten, waren jedoch bezüglich Schweregrad und Prognose der Krebserkrankungen nicht mit den Tumorpatienten aus den großen NMH-Studien CLOT und CATCH vergleichbar, sodass die Übertragbarkeit der Post-hoc-Analysen auf den klinischen Behandlungsalltag trotz eines vielversprechenden indirekten Vergleiches bezweifelt werden musste $(7,18)$.

Mittlerweile sind die Ergebnisse von zwei prospektiven, randomisierten Studien zum Einsatz von DOAKs bei Patienten mit tumorassoziierter VTE verfügbar, die vor dem Hintergrund aktueller Leitlinienempfehlungen einer genaueren Betrachtung bedürfen.

\section{HOKUSAI VTE Cancer}

Ende 2017 wurden die Ergebnisse der HOKUSAI VTE Cancer-Studie publiziert (19). Es handelte sich um eine prospektive, randomisierte, offene Studie mit verblindeter Endpunktevaluation (PROBE-Design), in die erwachsene Patienten mit akuter VTE und aktiver oder in den letzten zwei Jahren diagnostizierter Krebserkrankung eingeschlossen werden konnten (20). Eine aktive Krebserkrankung lag vor, wenn mindestens eines der folgenden drei Kriterien erfüllt war:

- Tumordiagnose oder -therapie in den letzten 6 Monaten

- Rezidiviertes, lokal fortgeschrittenes oder metastasiertes Tumorstadium

- Hämatologische Neoplasie in nicht kompletter Remission

Basalzell- oder Plattenepithelkarzinome der Haut wurden nicht berücksichtigt. Somit war die Definition einer aktiven Krebserkrankung in HOKUSAI VTE Cancer mit derjenigen aus den beiden großen NMHStudien CLOT und CATCH vergleichbar (Tabelle 1). Neben einer symptomatischen VTE konnten die Patienten auch eine unerwartet entdeckte, inzidentelle proximale
TVT oder LAE in segmentalen oder größeren Pulmonalarterien aufweisen.

Die Patienten erhielten entweder Dalteparin nach dem CLOT-Protokoll (1 x $200 \mathrm{IE}$ Anti-Xa/kg s.c. pro Tag über 30 Tage gefolgt von 1 x 150 IE Anti-Xa/kg s.c. pro Tag) oder Edoxaban 1 x 60 mg/Tag im Anschluss an eine therapeutisch dosierte parenterale Antikoagulation mit $\mathrm{NMH}$ über mindestens fünf Tage. Die EdoxabanDosis wurde nach den üblichen Kriterien auf 1 x 30 mg/Tag reduziert: KreatininClearance 30-50 ml/min, Körpergewicht $\leq 60 \mathrm{~kg}$ oder Begleittherapie mit einem starken P-Glykoprotein-Inhibitor. Die Randomisierung der Patienten erfolgte stratifiziert nach Blutungsrisiko und Notwendigkeit einer Edoxaban-Dosisreduktion. Alle Patienten sollten über mindestens sechs Monate, nach Möglichkeit jedoch über zwölf Monate behandelt werden. Kombinierter primärer Studienendpunkt war das Erstauftreten einer rezidivierten VTE oder einer schweren Blutung über den zwölfmonatigen Beobachtungszeitraum. Die Studie war auf Nichtunterlegenheit ausgelegt.

Von den 1.050 randomisierten Patienten erhielten 522 Edoxaban, 524 Dalteparin und vier Patienten keine Studienmedikation. Das mittlere Alter betrug in beiden Gruppen 64 Jahre. Jeweils etwa zwei Drittel der Patienten hatten als Indexereignis eine symptomatische VTE bzw. eine LAE mit oder ohne begleitende TVT. Eine aktive Krebserkrankung lag bei $98 \%$ und ein metastasiertes Tumorstadium bei $53 \%$ der Patienten vor. Die mediane Behandlungsdauer war im Dalteparin-Arm mit 184 Tagen um etwa einen Monat kürzer als im Edoxaban-Arm (211 Tage). Dieser Unterschied war statistisch signifikant $(\mathrm{P}=0,0143)$. Eine Therapie über die gesamten zwölf Monate oder bis zum Studienende erhielten 38,3\% der mit der Edoxaban und 29,4 \% der mit Dalteparin behandelten Patienten ( $\triangleright$ Abb. 2).

Bezüglich der eingeschlossenen Malignome hatten jeweils $11 \%$ der Patienten eine hämatologische Neoplasie. Die häufigsten soliden Tumorentitäten waren kolorektale, hepatobiliäre, gynäkologische, Bronchial-, Pankreas- und Mammakarzinome sowie Tumore des Urogenital- und oberen Gastrointestinal(GI-)Traktes.

Der kombinierte primäre Studienendpunkt aus VTE-Rezidiv und schwerer Blutung über den Beobachtungszeitraum von zwölf Monaten trat bei 12,8 \% der Patienten in der Edoxaban-Gruppe und bei 13,5 \% der Patienten in der DalteparinGruppe auf, entsprechend einer Hazard Ratio (HR) von 0,97 (95\%-Konfidenzintervall [KI] 0,70-1,36; $\mathrm{P}=0,006$ für Nichtunterlegenheit). Konsistente Ergebnisse wurden beobachtet, wenn nur die ersten sechs Monate oder nur die Patienten mit protokollgerechter Behandlung berücksichtigt wurden. Beide Subanalysen waren präspezifiziert. Somit hat HOKUSAI VTE Cancer das primäre Studienziel erreicht, wonach bei Patienten mit tumorassoziierter VTE oral verabreichtes Edoxaban einer parenteralen Antikoagulation mit Dalteparin bezüglich des klinischen Nettonutzens nicht unterlegen ist.
Abb. 2

Therapiepersistenz in HOKUSAI VTE Cancer: Gezeigt ist der Anteil der Patienten im Edoxaban- und Dalteparin-Arm, der zum jeweiligen Zeitpunkt noch die zugeordnete Studienmedikation erhielt $(R=$ Randomisierung). Daten aus Raskob et al. (19).

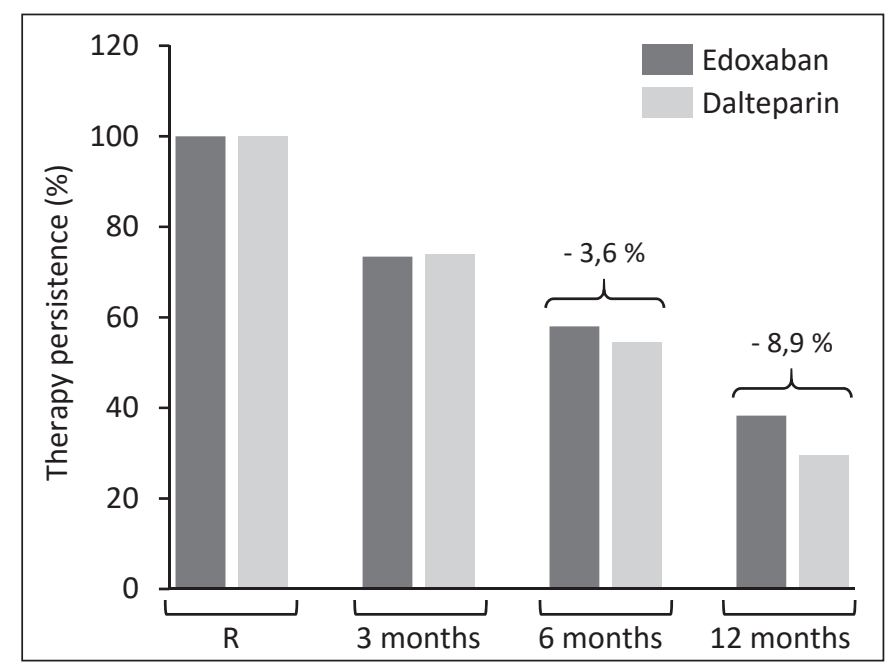


Tab. 1 Randomisierte kontrollierte Studien zum Vergleich NMH gegenüber VKA und DOAKs in der Therapie der tumorassoziierten VTE. NMH = Niedermolekulares Heparin; VKA = Vitamin K-Antagonist; $D O A K=$ Direktes orales Antikoagulanz; VTE = Venöse Thromboembolie; $T V T=$ Tiefe Venenthrombose; $L A E=$ Lungenarterienembolie; SAW = Standardabweichung; $H R=$ Hazard Ratio; 95\%-KI = 95\%-Konfidenzintervall.

\begin{tabular}{|c|c|c|c|c|}
\hline & \multicolumn{2}{|l|}{ NMH vs. VKA } & \multicolumn{2}{|l|}{ DOAK vs. NMH } \\
\hline & $\begin{array}{l}\text { CLOT } \\
\text { (Lee et al. } 2003 \text { [9]) }\end{array}$ & $\begin{array}{l}\text { CATCH } \\
\text { (Lee et al. } 2015 \text { [10]) }\end{array}$ & $\begin{array}{l}\text { HOKUSAI VTE Cancer } \\
\text { (Raskob et al. } 2018 \text { [19]) }\end{array}$ & $\begin{array}{l}\text { SELECT-D } \\
\text { (Young et al. } 2018 \text { [21]) }\end{array}$ \\
\hline \multicolumn{5}{|l|}{ Einschlusskriterien } \\
\hline $\begin{array}{l}\text { Tumor- } \\
\text { erkrankung }\end{array}$ & $\begin{array}{l}\text { Jede Tumorerkrankunga, die } \\
\text { innerhalb der letzten } 6 \text { Mo- } \\
\text { nate vor Studieneinschluss } \\
\text { diagnostiziert und/oder the- } \\
\text { rapiert wurde, sowie jede } \\
\text { bei Studieneinschluss rezi- } \\
\text { divierte oder metastasierte } \\
\text { Tumorerkrankung }\end{array}$ & $\begin{array}{l}\text { Jede Tumorerkrankung }{ }^{\text {a }} \text {, die in- } \\
\text { nerhalb der letzten } 6 \text { Monate } \\
\text { vor Studieneinschluss diagnosti- } \\
\text { ziert und/oder therapiert wurde, } \\
\text { jede bei Studieneinschluss rezi- } \\
\text { divierte, lokal fortgeschrittene } \\
\text { oder metastasierte Tumorer- } \\
\text { krankung sowie jede sich nicht } \\
\text { in kompletter Remission befin- } \\
\text { dende hämatologische Neopla- } \\
\text { sie }\end{array}$ & $\begin{array}{l}\text { Jede aktive oder innerhalb der } \\
\text { letzten zwei Jahre vor Studienein- } \\
\text { schluss diagnostizierte Tumorer- } \\
\text { krankunga; aktive Tumorerkran- } \\
\text { kung definiert als Tumorerkran- } \\
\text { kung, die innerhalb der letzten } 6 \\
\text { Monate vor Studieneinschluss di- } \\
\text { agnostiziert und/oder therapiert } \\
\text { wurde, jede bei Studieneinschluss } \\
\text { rezidivierte, lokal fortgeschrittene } \\
\text { oder metastasierte Tumorerkran- } \\
\text { kung sowie jede sich nicht in } \\
\text { kompletter Remission befindende } \\
\text { hämatologische Neoplasie }\end{array}$ & $\begin{array}{l}\text { Jede Tumorerkrankung }{ }^{\text {a }} \text {, die in- } \\
\text { nerhalb der letzten } 6 \text { Monate } \\
\text { vor Studieneinschluss diagnosti- } \\
\text { ziert und/oder therapiert wurde, } \\
\text { jede bei Studieneinschluss rezi- } \\
\text { divierte oder metastasierte Tu- } \\
\text { morerkrankung sowie jede sich } \\
\text { nicht in kompletter Remission } \\
\text { befindende hämatologische } \\
\text { Neoplasie }\end{array}$ \\
\hline Index-VTE & $\begin{array}{l}\text { Neudiagnostizierte sympto- } \\
\text { matische proximale TVT } \\
\text { und/oder LAE }\end{array}$ & $\begin{array}{l}\text { Neudiagnostizierte symptomati- } \\
\text { sche proximale TVT und/oder } \\
\text { LAE }\end{array}$ & $\begin{array}{l}\text { Neudiagnostizierte symptomati- } \\
\text { sche oder inzidentelle proximale } \\
\text { TVT und/oder LAE }\end{array}$ & $\begin{array}{l}\text { Symptomatische proximale TVT } \\
\text { und/oder symptomatische oder } \\
\text { inzidentelle LAE }\end{array}$ \\
\hline Studienarme & $\begin{array}{l}\text { Dalteparin } 200 \text { IE/kg (maxi- } \\
\text { mal } 18.000 \text { IE/Tag) für } 1 \\
\text { Monat, anschließend } 150 \\
\text { IE/kg vs. VKA (INR-Zielbe- } \\
\text { reich 2-3) initial überlap- } \\
\text { pend mit Dalteparin } 200 \text { IE/ } \\
\text { kg für 5-7 Tage }\end{array}$ & $\begin{array}{l}\text { Tinzaparin } 175 \text { IE/kg vs. VKA } \\
\text { (INR-Zielbereich 2-3) initial } \\
\text { überlappend mit Tinzaparin } 175 \\
\text { IE/kg für 5-10 Tage }\end{array}$ & $\begin{array}{l}\text { NMH in therapeutischer Dosie- } \\
\text { rung für } \geq 5 \text { Tage, anschließend } \\
\text { Edoxaban } 60 \mathrm{mg} / \mathrm{Tag}^{\mathrm{c}} \text { vs. Dalte- } \\
\text { parin } 200 \mathrm{IE} / \mathrm{kg} \text { (maximal } \\
18.000 \mathrm{IE} / \mathrm{Tag} \text { ) für } 30 \text { Tage, an- } \\
\text { schließend } 150 \mathrm{IE} / \mathrm{kg}^{\mathrm{d}}\end{array}$ & $\begin{array}{l}\text { Rivaroxaban } 2 \text { × } 15 \mathrm{mg} / \mathrm{Tag} \text { für } \\
3 \text { Wochen, anschließend } 20 \mathrm{mg} / \\
\text { Tage }^{\mathrm{e}} \text { vs. Dalteparin } 200 \mathrm{IE} / \mathrm{kg} \\
\text { (maximal } 18.000 \mathrm{IE} / \mathrm{Tag} \text { ) für } 30 \\
\text { Tage, anschließend } 150 \mathrm{IE} / \mathrm{kg}^{f}\end{array}$ \\
\hline Therapiedauer & 6 Monate & 6 Monate & 6-12 Monate & 6 Monate \\
\hline $\begin{array}{l}\text { Zeitpunkt der } \\
\text { Rekrutierung }\end{array}$ & 1999-2001 & 2010-2013 & 2015-2016 & 2013-2016 \\
\hline $\begin{array}{l}\text { Anzahl an Patien- } \\
\text { ten }\end{array}$ & 676 & 900 & 1.046 & 406 \\
\hline $\begin{array}{l}\text { Alter (Jahre), } \\
\text { Mittelwert } \pm \\
\text { SAW oder Medi- } \\
\text { an (Spannbreite) }\end{array}$ & $62 \pm 12$ bzw. $63 \pm 13$ & $59,7 \pm 12,7$ bzw. $58,8 \pm 12,5$ & $64,3 \pm 11$ bzw. $63,7 \pm 11,7$ & $67(22-87)$ \\
\hline \multicolumn{5}{|l|}{$\begin{array}{l}\text { ECOG-Stadium, } \\
\text { Anzahl (Prozent) }\end{array}$} \\
\hline $0-1$ & $428(63)$ & $691(77)$ & $792(76)$ & $304(76)$ \\
\hline 2 & $240(36)$ & $209(23)$ & 247 (24) & 95 (24) \\
\hline 3 & $8(1)$ & & & \\
\hline $\begin{array}{l}\text { Hämatologische } \\
\text { Neoplasie, } \\
\text { Anzahl (Prozent) }\end{array}$ & $70(10)$ & $94(10)$ & $111(11)$ & $31(8)$ \\
\hline $\begin{array}{l}\text { Metastasierte Tu- } \\
\text { morerkrankung, } \\
\text { Anzahl (Prozent) }\end{array}$ & $455(67)$ & $492(55)$ & $554(53)$ & $236(58)$ \\
\hline
\end{tabular}


Tab. 1 Fortsetzung.

\begin{tabular}{|c|c|c|c|c|c|c|c|c|c|c|c|c|}
\hline & \multicolumn{6}{|c|}{ NMH vs. VKA } & \multicolumn{6}{|c|}{ DOAK vs. NMH } \\
\hline & \multicolumn{3}{|c|}{$\begin{array}{l}\text { CLOT } \\
\text { (Lee et al. } 2003 \text { [9]) }\end{array}$} & \multicolumn{3}{|c|}{$\begin{array}{l}\text { CATCH } \\
\text { (Lee et al. } 2015 \text { [10]) }\end{array}$} & \multicolumn{3}{|c|}{$\begin{array}{l}\text { HOKUSAI VTE Cancer } \\
\text { (Raskob et al. } 2018 \text { [19]) }\end{array}$} & \multicolumn{3}{|c|}{$\begin{array}{l}\text { SELECT-D } \\
\text { (Young et al. } 2018 \text { [21]) }\end{array}$} \\
\hline $\begin{array}{l}\text { Tumortherapie } \\
\text { bei Studienein- } \\
\text { schluss, } \\
\text { Anzahl (Prozent) }\end{array}$ & \multicolumn{3}{|l|}{$525(78)$} & \multicolumn{3}{|l|}{$476(53)$} & \multicolumn{3}{|l|}{$757(72)^{g}$} & \multicolumn{3}{|l|}{$282(69)$} \\
\hline \multirow{2}{*}{$\begin{array}{l}\text { Inzidentelle } \\
\text { Index-VTE, } \\
\text { Anzahl (Prozent) }\end{array}$} & & & & & & & $340(33)$ & & & $213(52)^{h}$ & & \\
\hline & $\begin{array}{l}\text { Dalte- } \\
\text { parin } \\
(n=336)\end{array}$ & $\begin{array}{l}\text { VKA } \\
(n=336)\end{array}$ & $\begin{array}{l}\text { HR } \\
(95 \%- \\
\mathrm{KI})\end{array}$ & $\begin{array}{l}\text { Tinzapa- } \\
\text { rin } \\
(n=449)\end{array}$ & $\begin{array}{l}\text { VKA } \\
(n=451)\end{array}$ & $\begin{array}{l}\text { HR } \\
(95 \%-K I)\end{array}$ & $\begin{array}{l}\text { Edoxa- } \\
\text { ban } \\
(n=522)\end{array}$ & $\begin{array}{l}\text { Daltepa- } \\
\text { rin } \\
(n=524)\end{array}$ & $\begin{array}{l}\text { HR } \\
(95 \%-K I)\end{array}$ & $\begin{array}{l}\text { Rivaroxa- } \\
\text { ban } \\
(n=203)\end{array}$ & $\begin{array}{l}\text { Daltepa- } \\
\text { rin } \\
(n=203)\end{array}$ & $\begin{array}{l}\text { HR } \\
(95 \%-K I)\end{array}$ \\
\hline $\begin{array}{l}\text { VTE-Rezidiv, } \\
\text { Anzahl (Prozent) }\end{array}$ & $27(8)$ & $53(16)$ & $\begin{array}{l}0,48 \\
(0,30- \\
0,77)\end{array}$ & $31(7)$ & $45(10)$ & $\begin{array}{l}0,65 \\
(0,41-1 \\
03)\end{array}$ & $41(8)$ & 59 (11) & $\begin{array}{l}0,71 \\
(0,48-1 \\
06)\end{array}$ & $8(4)$ & $18(9)$ & $\begin{array}{l}0,43 \\
(0,19-0, \\
99)\end{array}$ \\
\hline $\begin{array}{l}\text { Schwere } \\
\text { Blutung, } \\
\text { Anzahl (Prozent) }\end{array}$ & $19(6)$ & $12(4)$ & & $12(3)$ & $11(2)$ & $\begin{array}{l}0,89 \\
(0,40-1 \\
99)\end{array}$ & $36(7)$ & $21(4)$ & $\begin{array}{l}1,77 \\
(1,03-3, \\
04)\end{array}$ & $11(5)$ & $6(3)$ & $\begin{array}{l}1,83 \\
(0,68-4, \\
96)\end{array}$ \\
\hline $\begin{array}{l}\text { Klinisch relevan- } \\
\text { te nichtschwere } \\
\text { Blutung, } \\
\text { Anzahl (Prozent) }\end{array}$ & & & & 49 (11) & $69(15)$ & $\begin{array}{l}0,58 \\
(0,40-0, \\
84)\end{array}$ & $76(15)$ & $58(11)$ & $\begin{array}{l}1,38 \\
(0,98-1 \\
94)\end{array}$ & $25(12)$ & $7(3)$ & $\begin{array}{l}3,76 \\
(1,63-8, \\
69)\end{array}$ \\
\hline $\begin{array}{l}\text { Gesamt- } \\
\text { mortalität, } \\
\text { Anzahl (Prozent) }\end{array}$ & $\begin{array}{l}130 \\
(39)\end{array}$ & $\begin{array}{l}136 \\
(41)\end{array}$ & & $150(33)$ & $138(31)$ & $\begin{array}{l}1,08 \\
(0,85-1 \\
36)\end{array}$ & $206(40)$ & $192(37)$ & $\begin{array}{l}1,12 \\
(0,92-1 \\
37)\end{array}$ & 48 (24) & $56(28)$ & \\
\hline
\end{tabular}

a Ausgenommen Basaliome und nichtmelanomatöser Hautkrebs.

b Bei 1.024 von insgesamt 1.046 eingeschlossenen Patienten (98\%) lag eine aktive Tumorerkrankung vor.

c Dosisreduktion auf Edoxaban $30 \mathrm{mg} /$ Tag falls Kreatinin-Clearance 30-50 ml/min, Körpergewicht $\leq 60 \mathrm{~kg}$ oder gleichzeitige Einnahme von starken P-Glykoprotein-Inhibitoren.

d Vorübergehende Dosisreduktion von Dalteparin falls Thrombozyten $<100 \times 10^{9} /$.

e Therapieunterbrechung falls Thromboyzten $<50 \times 10^{9} /$ sowie Dosisanpassung oder Therapieunterbrechung bei eingeschränkter Nierenfunktion.

f Vorübergehende Dosisanpassung oder Therapieunterbrechung falls Thrombozytopenie oder signifikant eingeschränkte Nierenfunktion.

$g$ Tumortherapie innerhalb der letzten 4 Wochen vor Studieneinschluss.

h Bei 6 Patienten lag neben einer inzidentellen LAE eine TVT vor.

Wenn die Komponenten des primären Endpunkts einzeln betrachtet werden, traten VTE-Rezidive, insbesondere symptomatische Ereignisse und isolierte TVTs, in der Edoxaban-Gruppe seltener auf als in der Dalteparin-Gruppe (6,5\% vs. 10,3\%). Dagegen waren schwere Blutungen, insbesondere solche aus dem oberen GI-Trakt, in der Edoxaban-Gruppe häufiger als in der Dalteparin-Gruppe (6,3\% vs. $3,2 \%)$.

Als sekundäre Studienendpunkte wurden alle VTE-Rezidive und Blutungsereignisse über den zwölfmonatigen Beobachtungszeitraum ausgewertet. Auch in dieser Analyse traten VTE-Rezidive mit 7,9\% numerisch seltener im Edoxaban- als im DalteparinArm (11,3 \%) auf, entsprechend einer HR von 0,71 (95 \%-KI 0,48-1,06; $\mathrm{P}=0,09)$. Die verminderte VTE-Rezidivrate war vor allem durch eine geringere Anzahl an wiederkehrenden TVTs in der Edoxaban-Gruppe bedingt (HR 0,56; 95 \%-KI 0,32-0,97), während die Anzahl wiederkehrender LAEs zwischen den Behandlungsgruppen vergleichbar war (HR 1,00; 95 \%-KI 0,59-1,69). Bezüglich der sekundären Sicherheitsendpunkte wurden unter der Antikoagulation mit Edoxaban (6,9 \%) signifikant mehr schwere Blutungsereignisse beobachtet als unter der Antikoagulation mit Dalteparin (4,0\%), entsprechend einer HR von 1,77 (95 \%-KI 1,03-3,04; $\mathrm{P}=0,04$ ). Auch die Kombination aus schwerer und klinisch relevanter nichtschwerer Blutung war im Edo- xaban- häufiger als im Dalteparin-Arm (18,6 \% vs. $13,9 \%$ ), während die Gesamtmortalität mit 39,5\% und 36,6 \% in beiden Behandlungsgruppen vergleichbar war.

In einer präspezifizierten Analyse wurden die schweren Blutungsereignisse nach ihrer medizinischen Dringlichkeit vier verschiedenen Kategorien zugeordnet. Eine schwere Blutung lag vor, wenn mindestens eines der folgenden Kriterien erfüllt war:

- Tödlicher Ausgang

- Transfusion von zwei oder mehr Erythrozytenkonzentraten

- Abfall des Hb-Wertes um $\geq 2 \mathrm{~g} / \mathrm{dl}$

- Blutung in einer kritischen Lokalisation (z. B. retroperitoneale, intrakranielle, intraokuläre oder intraartikuläre Blutung) 
Schwere Blutungen der Kategorie 4 waren solche Blutungen, die bereits vor der Krankenhausaufnahme oder unmittelbar danach zum Tode führten, während schwere Blutungen der Kategorie 3 einen medizinischen Notfall darstellten (z. B. GI-Blutung mit hämodynamischer Instabilität oder intrakranielle Blutung mit neurologischer Symptomatik). Schwere Blutungen der Kategorie 1 waren mit keiner medizinischen Notfallsituation verbunden, wohingegen schwere Blutungen der Kategorie 2 keiner anderen Kategorie zugeordnet werden konnten. Schwere Blutungen der Kategorie 3 oder 4 traten bei $2,3 \%(12 / 522)$ der mit Edoxaban und bei 2,5 \% (13/524) der mit Dalteparin behandelten Patienten auf. Da Blutungsereignisse der Kategorie 1 nicht beobachtet wurden, war die Zunahme schwerer Blutungen in der EdoxabanGruppe vor allem durch Blutungen der Kategorie 2 bedingt, die bei 24/522 (4,6\%) vs. $8 / 524$ (1,5\%) Patienten auftaten. Eine Analyse der Sicherheitskomponente des primären Studienendpunktes legt nahe, dass es sich hierbei vor allem um obere GI-Blutungen gehandelt hat.
Die Patienten-Randomisierung in HOKUSAI VTE Cancer erfolgte stratifiziert nach dem Blutungsrisiko. Hierfür wurden vorab die folgenden sechs Risikofaktoren definiert:

- Operativer Eingriff vor $\leq 2$ Wochen

- Einnahme von Plättcheninhibitoren

- Primärer Hirntumor oder Hirnmetastasen

- Lokal fortgeschrittene oder metastasierte Tumorerkrankung

- GI- oder urothelialer Tumor entweder in situ oder diagnostiziert vor $\leq 6$ Monaten

- Therapie mit dem VEGF-Antikörper Bevacizumab vor $\leq 6$ Wochen

Zum Zeitpunkt der Randomisierung hatten lediglich 17,6\% der Patienten in beiden Gruppen kein Blutungsrisiko, während bei den übrigen 82,4 \% der Patienten mindestens einer der genannten sechs Risikofaktoren vorlag. Eine Subgruppenanalyse zeigt, dass Patienten mit GI-Tumoren unter Einnahme von Edoxaban ein signifikant höheres Risiko für schwere Blutungen hatten als unter einer Therapie mit Dalteparin
( Abb. 3): Schwere Blutungen traten bei 18/136 (13,2 \%) der mit Edoxaban und bei 3/125 (2,4 \%) der mit Dalteparin behandelten Patienten auf $(\mathrm{P}=0,017)$.

Bezüglich der sekundären Wirksamkeits- und Sicherheitsendpunkte schienen Patienten, die zum Zeitpunkt der Randomisierung kein Blutungsrisiko aufwiesen, von einer Therapie mit Edoxaban zu profitieren. Bei diesen Patienten lag die VTE-Rezidivrate (Edoxaban vs. Dalteparin) bei 3,3\% vs. $13,0 \%$ und die Rate für schwere Blutungen bei $4,3 \%$ vs. 5,4 \%. Dagegen war die Wirksamkeit bei Patienten mit mindestens einem Blutungsrisikofaktor zum Zeitpunkt der Randomisierung in beiden Behandlungsarmen vergleichbar ( $8,8 \%$ vs. $10,9 \%)$ bei numerisch mehr schweren Blutungen in der Edoxaban-Gruppe (7,4 \% vs. 3,7 \%). Es bedarf jedoch der Anmerkung, dass es sich hierbei bestenfalls um eine explorative, Hypothesen generierende Analyse handelt, da das Blutungsrisiko zum Zeitpunkt der Randomisierung weder auf den Wirksamkeits(P für Interaktion 0,064) noch auf den Sicherheitsendpunkt (P für Interaktion 0,165) einen signifikanten Einfluss hatte.

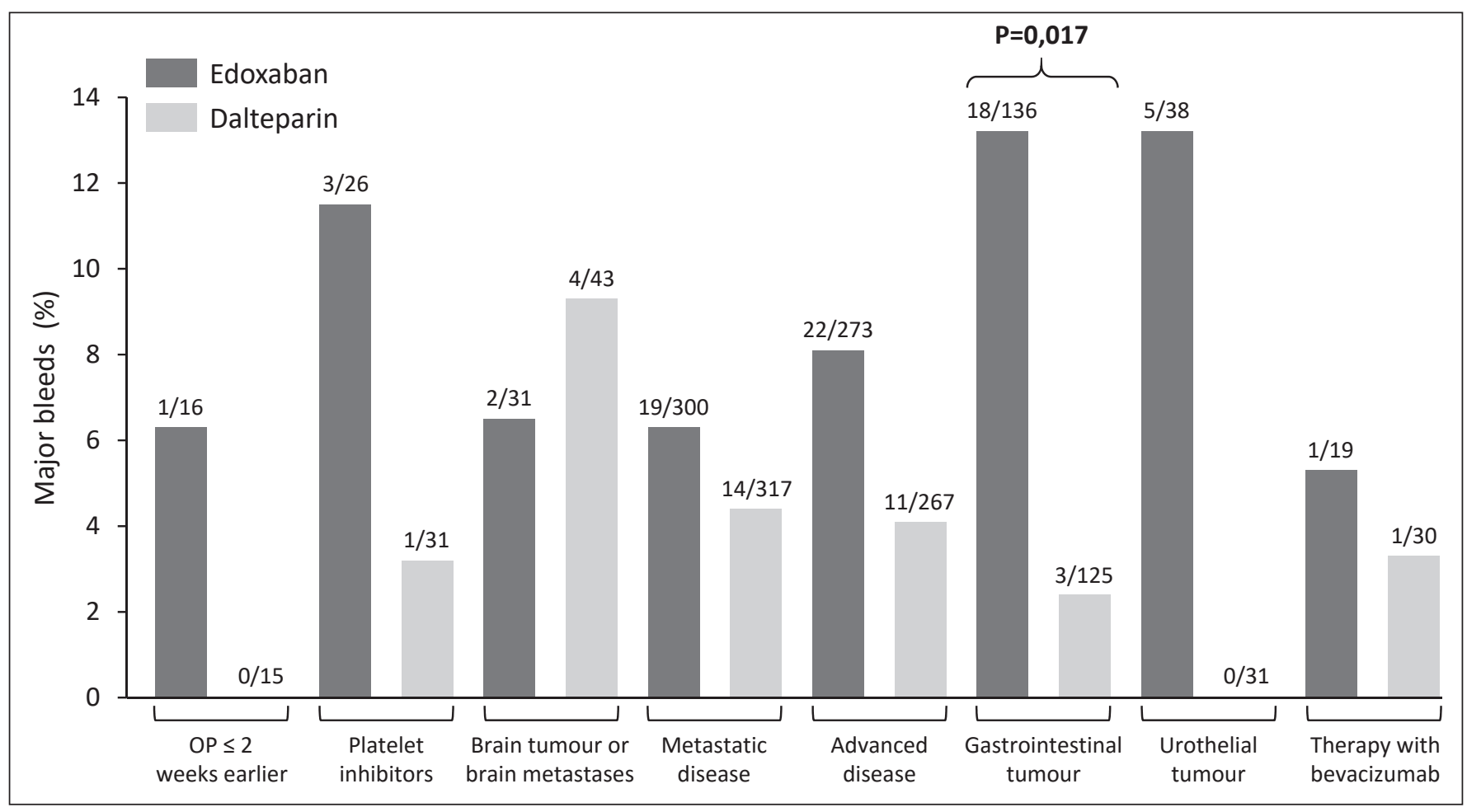

Abb. 3 Subgruppenanalyse der Häufigkeit schwerer Blutungen in HOKUSAI VTE Cancer. Gezeigt ist der Anteil der Patienten mit schweren Blutungen im Edoxaban- und Dalteparin-Arm in Abhängigkeit vom Vorliegen eines präspezifizierten Risikofaktors. Die Analyse basiert auf der Sicherheitspopulation (alle Patienten, die randomisiert wurden und mindestens eine Dosis der Studienmedikation erhielten). Der P-Wert zeigt eine signifikante Interaktion zwischen dem Risikofaktor "gastrointestinaler Tumor" und dem Auftreten schwerer Blutungen in Abhängigkeit von der zugeordneten Therapie an. Daten aus Raskob et al. (19). 


\section{SELECT-D}

Seit diesem Jahr im Volltext verfügbar sind die Ergebnisse der SELECT-D-Studie, in der das NMH Dalteparin nach dem CLOTProtokoll mit dem direkten oralen Faktor Xa-Inhibitor Rivaroxaban $(2 \times 15 \mathrm{mg} /$ Tag für drei Wochen gefolgt von 1 x $20 \mathrm{mg} /$ Tag für insgesamt sechs Monate) bei Patienten mit tumorassoziierter VTE verglichen wurde (21). Es handelte sich um eine prospektive, randomisierte, offene Pilotstudie an 58 Zentren in Großbritannien und Nordirland. Primäres Studienziel war es, die Rate von VTE-Rezidiven unter einer Antikoagulation mit Rivaroxaban als Grundlage für eine nachfolgende Phase 3-Studie zu ermitteln. Im Anschluss an die initiale Randomisierung (Dalteparin vs. Rivaroxaban) sah die Studie nach sechs Monaten eine zweite Randomisierung vor: Patienten mit LAE als Indexereignis oder einer relevanten Restthrombose sollten für weitere sechs Monate verblindet entweder Placebo oder Rivaroxaban 1 x 20 mg/Tag erhalten. Bisher sind nur die Ergebnisse für den ersten Teil der Studie verfügbar.

Ursprünglich sollten in SELECT-D 530 Patienten mit aktiver Krebserkrankung und symptomatischer proximaler TVT, symptomatischer LAE oder inzidenteller LAE eingeschlossen werden. Aufgrund langsamer Rekrutierung wurde die Fallzahl im Verlauf der Studie auf 400 Patienten (200 Patienten pro Behandlungsarm) korrigiert. Die Randomisierung erfolgte stratifiziert nach Tumorstadium, Thrombozytenzahl ( $\leq 350$ vs. $\left.>350 \times 10^{9} / 1\right)$, Typ der VTE (symptomatisch vs. inzidentelle LAE) und Thrombogenität des Primärtumors (hohes vs. niedriges VTE-Rezidivrisiko). In SELECT-D war eine therapeutische Antikoagulation über 96 Stunden vor Studieneinschluss möglich, während eine Begleittherapie mit starken Inhibitoren oder Induktoren von CYP3A4 oder P-Glykoprotein nicht erlaubt war.

Zwischen September 2013 und Dezember 2016 wurden insgesamt 406 Patienten (203 Patienten pro Behandlungsarm) in die Studie eingeschlossen. Das mediane Alter lag in beiden Gruppen bei 67 Jahren. Jeweils $58 \%$ der Patienten hatten ein metastasiertes Tumorstadium sowie jeweils etwas mehr als die Hälfte eine inzidentelle LAE als Indexereignis. Vergleichbar zu HOKUSAI VTE Cancer hatten in SELECT-D etwa $90 \%$ der Patienten eine solide Tumorerkrankung und nur etwa $10 \%$ eine hämatologische Neoplasie (z. B. CLL, Myelom oder Lymphom). Die mediane Behandlungsdauer lag bei 5,8 Monaten in der Dalteparin- und bei 5,9 Monaten in der Rivaroxaban-Gruppe. Der Anteil an Patienten, die mindestens eine ausgelassene Dosis angaben, war unter der Therapie mit dem DOAK (27 \%) geringfügig höher als unter der Therapie mit dem NMH (22\%).

Über den sechsmonatigen Behandlungszeitraum trat ein VTE-Rezidiv bei 8 Patienten in der Rivaroxaban- und bei 18 Patienten in der Dalteparin-Gruppe auf, entsprechend einer kumulativen VTE-Rezidivrate (Rivaroxaban vs. Dalteparin) von $4 \%$ (95 \%-KI 2-9 \%) vs. $11 \%$ (95\%-KI 7-16\%) und einer HR von 0,43 (95\%-KI 0,19-0,99). Bezüglich der VTE-Rezidivereignisse wurden insbesondere weniger TVTs (3 vs. 7) und weniger inzidentelle LAEs (1 vs. 6) in der Rivaroxaban-Gruppe beobachtet. Prädiktive Risikofaktoren für ein VTE-Rezidiv waren die Lokalisation des Primärtumors (HR 5,6 für Magen-/ Pankreaskarzinome und HR 2,7 für Lymphome, gynäkologische Malignome oder
Lungen-/Blasenkarzinome vs. andere $\mathrm{Tu}-$ more) sowie der Typ der Index VTE (HR 2,8 für symptomatische VTE vs. inzidentelle LAE).

Schwere Blutungen traten bei 11 Patienten in der Rivaroxaban- und bei 6 Patienten in der Dalteparin-Gruppe auf, entsprechend einer kumulativen Blutungsrate (Rivaroxaban vs. Dalteparin) von $6 \%$ (95 \%-KI 3-11 \%) vs. $4 \%$ (95 \%-KI 2-8 \%) und einer HR von 1,83 (95 \%-KI 0,68-4,96). Bei den schweren Blutungen handelte es sich vor allem um Blutungen aus dem oberen oder unteren GI-Trakt. Insbesondere Patienten mit (gastro-)ösophagealen Tumoren hatten unter der Einnahme von Rivaroxaban ein erhöhtes Blutungsrisiko. Bei diesen Patienten wurden schwere Blutungen bei 1/19 (5\%) in der Dalteparin-Gruppe und bei 4/11 (36\%) in der Rivaroxaban-Gruppe beobachtet. Auf der Basis einer Zwischenauswertung nach den ersten 220 Patienten wurden Patienten mit diesen Tumorlokalisationen von der weiteren Rekrutierung ausgeschlossen.

Die kumulative Rate für klinisch relevante nichtschwere Blutungen lag bei $4 \%$ (7 Ereignisse) in der Dalteparin- und bei 13 \% (25 Ereignisse) in der RivaroxabanGruppe, entsprechend einer HR von

\section{Abb. 4}

Häufigkeit klinisch relevanter Blutungen bei Patienten mit gastrointestinalen Tumoren in SELECT-D. Gezeigt ist die Häufigkeit von schweren und klinisch relevanten nichtschweren (KRNS) Blutungen bei Patienten, die zum Zeitpunkt der Randomisierung unter einem Tumor im Bereich der Speiseröhre oder des gastroösophagealen Übergangs $(n=30)$, des Magens $(n=11)$ oder des Dickdarms/Rektums $(n=102)$ litten. Daten aus Young et al. (21).

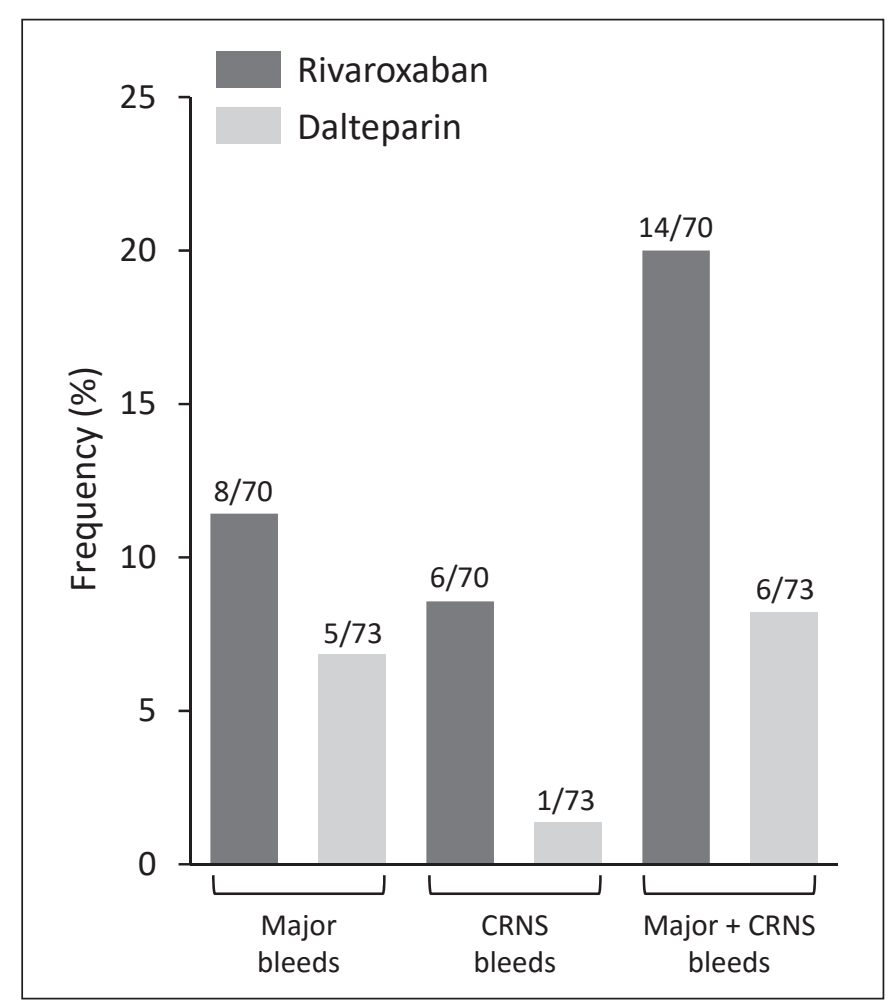


3,76 (95 \%-KI 1,63-8,69). Bei diesen Ereignissen handelte es sich vor allem um GIoder urotheliale Blutungen. Bezüglich des Gesamtüberlebens fand sich mit $70 \%$ (Dalteparin) und $75 \%$ (Rivaroxaban) kein Unterschied zwischen den Behandlungsgruppen.

In SELECT-D hatten insgesamt $143 \mathrm{~Pa}$ tienten einen Tumor im Bereich von Speiseröhre, gastroösophagealem Übergang, Magen, Dickdarm oder Enddarm. In dieser Subgruppe von Patienten mit GI-Tumoren wurden mehr klinisch relevante Blutungen in der Rivaroxaban- als in der DalteparinGruppe beobachtet ( Abb. 4).

\section{Metaanalyse HOKUSAI VTE Cancer und SELECT-D}

In einer Metaanalyse wurden die Ergebnisse der HOKUSAI VTE Cancer- und SELECT-D-Studie unter Berücksichtigung der ersten sechs Therapiemonate zusammengefasst (22). Danach fand sich im Vergleich zu Dalteparin bezüglich der Verhinderung von VTE-Rezidiven ein starker Trend zugunsten der beiden Faktor Xa-Inhibitoren (HR 0,65; 95 \%-KI 0,42-1,01) bei signifikanter Zunahme von schweren Blutungen (HR 1,74; 95 \%-KI 1,05-2,88). Auch klinisch relevante nichtschwere Blutungen traten unter der DOAK-Einnahme numerisch häufiger auf als unter der $\mathrm{NMH}$-Therapie mit einer HR von 2,31 (95\%-KI 0,85-6,28), während sich bezüglich der Gesamtsterblichkeit nach sechs Monaten zwischen den Behandlungsgruppen (DOAK vs. NMH) kein Unterschied zeigte (HR 1,03; 95 \%-KI 0,85-1,26).

\section{Diskussion und Fazit für die Praxis}

Auch wenn sich die Einnahmeschemata von Edoxaban und Rivaroxaban grundlegend unterscheiden, zeigen die beiden ersten großen randomisierten DOAK-Studien HOKUSAI VTE Cancer und SELECT-D konsistente Ergebnisse. Danach ist die DOAK-Einnahme zwar mit einer verbesserten Wirksamkeit, aber zugleich mit einem gesteigerten Blutungsrisiko assoziiert. Diese gegenläufigen Wirksamkeits- und Sicher- heitsdaten erschweren die Einordnung der Studienergebnisse in den klinischen Behandlungsalltag.

Die verbesserte Wirksamkeit von NMH gegenüber VKA gilt trotz der zuvor gemachten Anmerkungen bezüglich der Notwendigkeit einer optimalen INR-Einstellung als etabliert und stellt die Grundlage für die aktuellen Leitlinienempfehlungen dar. Dennoch wird bei Patienten mit aktiver Krebserkrankung die VTE-Rezidivrate unter einer Therapie mit NMH weiterhin als inakzeptabel hoch eingestuft. In CLOT lag die VTE-Rezidivrate unter Dalteparin nach sechs Monaten bei 8,0 \% (27/336), in HOKUSAI VTE Cancer bei 8,8 \% (46/524) und in SELECT-D bei 8,9 \% (18/208). Diese konsistenten Ereignisraten in den Dalteparin-Armen zeigen, dass die Studien bezüglich der eingeschlossenen Tumorpatienten vergleichbar sind, auch wenn zwischen der Rekrutierung der CLOT-Studie und der Rekrutierung der beiden DOAKStudien fast 15 Jahre liegen (Tabelle 1).

Als mögliche Erklärung für die verbesserte DOAK-Wirksamkeit wird mitunter die Reduktion der Dalteparin-Dosis auf 75-80 \% der Initialdosis nach 30 Tagen angeführt. Hieraus ergibt sich die Frage, ob ein Vergleich von Edoxaban mit Tinzaparin, das nach dem CATCH-Protokoll in therapeutischer Dosis über die gesamten sechs Monate verabreicht wird, zu ähnlichen Ergebnissen geführt hätte. Eine genauere Betrachtung des zeitlichen Auftretens der VTE-Rezidive in HOKUSAI VTE Cancer zeigt jedoch, dass die Ereigniskurven für diesen sekundären Wirksamkeitsendpunkt erst nach $>90$ Tagen divergieren, obwohl das thromboembolische Rezidivrisiko bei Tumorpatienten bekanntermaßen gerade in den ersten 1-2 Monaten nach Auftreten der Index-VTE besonders erhöht ist (1-3). Der Anteil der Patienten mit < 3 Monaten Therapie ist in HOKUSAI VTE Cancer mit 26,6 \% (Edoxaban) und 26,1 \% (Dalteparin) zwischen den beiden Behandlungsarmen nahezu identisch. Erst danach zeigt sich in der Dalteparin-Gruppe eine höhere Abbruchrate ( $\triangle$ Abb. 2). Unannehmlichkeiten mit der Medikamentenapplikation als Grund für einen permanenten Therapieabbruch wurden mehr als dreimal so häufig in der Dalteparin-Gruppe $(14,9 \%)$ als in der Edoxaban-Gruppe
$(4,0 \%)$ angegeben. Somit käme in HOKUSAI VTE Cancer prinzipiell eine geringere Therapiepersistenz als Ursache für die verminderte Wirksamkeit der NMH-Therapie in Frage. Nach dieser Interpretation wäre die DOAK-Einnahme effizienter, jedoch nicht unbedingt effektiver als die subkutanen NMH-Injektionen. Oder anders formuliert: Die verminderte VTE-Rezidivrate unter Edoxaban basiert weniger auf einer verbesserten pharmakodynamischen Wirkung als auf einem leichter umzusetzenden Therapiekonzept. Ein weiterer Grund könnte jedoch auch die geforderte Anpassung der Dalteparin-Dosis bei Thrombozytenwerten von $50-100 \times 10^{9} / 1$ sein, während die Einnahme von Edoxaban in der Standarddosierung bis zu einer Thrombozytenzahl von 50 x $10^{9} / 1$ erlaubt war.

Interessant ist die Beobachtung, dass in HOKUSAI VTE Cancer die Ereigniskurven für den sekundären Sicherheitsendpunkt „schwere Blutungen" bereits nach etwa zehn Tagen divergieren. $\mathrm{Zu}$ diesem Zeitpunkt war bereits bei einem Großteil der Patienten im Edoxaban-Arm die Umstellung auf das DOAK erfolgt: Die mediane Dauer der initialen NMH-Therapie lag bei fünf Tagen.

Aus diesen Anmerkungen ließe sich für den Praxisalltag die Handlungsempfehlung ableiten, bei einem Patienten mit tumorassoziierter VTE und mindestens einem der genannten sechs Blutungsrisikofaktoren die Antikoagulation über die ersten 4-12 Wochen zunächst mit NMH durchzuführen und in dieser Phase den Patienten besonders sorgfältig bezüglich etwaiger Blutungssymptome zu überwachen. Bei guter Verträglichkeit wäre dann die Umstellung auf Edoxaban möglich, zumal in HOKUSAI VTE Cancer die Dauer der initialen NMH-Therapie im Ermessen des Prüfarztes lag. Während Patienten ohne Blutungsrisiko über eine verbesserte Therapiepersistenz von der oralen Tabletteneinnahme profitieren, ist für Patienten mit Blutungsrisiko die langfristige $\mathrm{NMH}$-Therapie eine wirksame und sichere Option, wenn sie von den Vorteilen der parenteralen Medikamentenapplikation überzeugt sind und diese konsequent durchführen (23).

In SELECT-D kann die verminderte VTE-Rezidivrate unter Einnahme von Rivaroxaban nicht durch eine höhere Thera- 
pieabbruchrate in der NMH-Gruppe erklärt werden, da die mediane Behandlungsdauer in beiden Therapiearmen vergleichbar war. Hieraus ergibt sich die Hypothese, dass die hochdosierte therapeutische Antikoagulation mit dem direkten oralen Faktor Xa-Inhibitor über die ersten drei Wochen in einer besonders effektiven, nachhaltigen Kontrolle der paraneoplastischen Gerinnungsprozesse resultierte. Wichtig ist die Beobachtung, dass gerade diese initiale Therapiephase nicht mit einer dramatischen Zunahme an schweren Blutungen assoziiert war.

Vergleichbar zu HOKUSAI VTE Cancer hatten in SELECT-D insbesondere Patienten mit Tumoren im oberen oder unteren GI-Trakt unter der DOAK-Einnahme ein erhöhtes Blutungsrisiko ( Abb. 3 und 4). Diesbezüglich kommen entweder die intraluminal wachsenden Tumoren selbst oder therapieinduzierte Schleimhautläsionen im GI-Trakt, z. B. eine toxische Mukositis nach Behandlung mit 5-Fluorouracil, als Blutungsquelle in Frage. Diese Patienten scheinen nach heutigem Kenntnisstand für eine DOAK-Therapie ungeeignet, ebenso wie Patienten mit einem Urothelzellkarzinom der Harnblase: In SELECT-D hatten 6/10 (60 \%) Patienten mit letztgenannter Tumorentität unter Einnahme von Rivaroxaban eine klinisch relevante Blutung, während diese bei 1/4 (25 \%) Patienten in der Dalteparin-Gruppe beobachtet wurde. In HOKUSAI VTE Cancer traten schwere Blutungen (Edoxaban vs. Dalteparin) bei 5/38 (13 \%) vs. 0/31 (0 \%) Patienten mit urothelialen Tumoren auf ( Abb. 2).

HOKUSAI VTE Cancer und SELECT-D zeigen, dass bei Patienten mit tumorassoziierter VTE eine orale Antikoagulation mit Edoxaban oder Rivaroxaban als Alternative zur leitlinienkonformen NMHTherapie in Frage kommt. Diese Schlussfolgerung wird durch verschiedene kleinere Beobachtungsstudien bekräftigt (22). Eine sorgfältige Selektion unter Berücksichtigung von Patientenpräferenz und spezifischen Tumorcharakteristika ist jedoch erforderlich, um im Sinne einer individualisierten Therapie die jeweiligen Vor- und Nachteile oraler und parenteraler Antikoagulantien in einen optimalen klinischen Nettonutzen umzuwandeln.

\section{Interessenkonflikte}

MV hat Reiseunterstützung von den Firmen Bristol-Myers Squibb und LEO Pharma erhalten. JY erklärt keine Interessenkonflikte. FL hat Honorare für Beraterund/oder Vortragstätigkeit sowie Reiseunterstützung von den folgenden Firmen erhalten: Aspen, Bayer, Boehringer-Ingelheim, Bristol-Myers Squibb, Daiichi Sankyo, LEO Pharma, Pfizer und Sanofi.

\section{Ethische Richtlinien}

Für das Manuskript wurden keine Studien an Menschen oder Tieren durchgeführt.

\section{Literatur}

1. Levitan N, Dowlati A, Remick SC et al. Rates of initial and recurrent thromboembolic disease among patients with malignancy versus those without malignancy. Risk analysis using Medicare claims data. Medicine (Baltimore) 1999; 78(5): 285-291.

2. Prandoni P, Lensing AW, Piccioli A et al. Recurrent venous thromboembolism and bleeding complications during anticoagulant treatment in patients with cancer and venous thrombosis. Blood 2002 100(10): 3484-3488.

3. Monreal $M$, Falgá $C$, Valdés $M$ et al. Riete Investigators. Fatal pulmonary embolism and fatal bleeding in cancer patients with venous thromboembolism: findings from the RIETE registry. J Thromb Haemost. 2006 Sep;4(9):1950-1956.

4. Bauersachs R. [Prolonged Secondary Prevention After Venous Thromboembolism]. Dtsch Med Wochenschr 2018; 143(3): 137-142.

5. Lee AYY. Overview of VTE treatment in cancer according to clinical guidelines. Thromb Res 2018; 164(Suppl 1): S162-S167.

6. Carrier M, Cameron C, Delluc A, Castellucci L, Khorana AA, Lee AY. Efficacy and safety of anticoagulant therapy for the treatment of acute cancerassociated thrombosis: a systematic review and meta-analysis. Thromb Res 2014; 134(6): 1214-1219.

7. Posch F, Konigsbrugge O, Zielinski C, Pabinger I, Ay C. Treatment of venous thromboembolism in patients with cancer: A network meta-analysis comparing efficacy and safety of anticoagulants. Thromb Res 2015; 136(3): 582-589.

8. Kahale LA, Hakoum MB, Tsolakian IG et al. Anticoagulation for the long-term treatment of venous thromboembolism in people with cancer. Cochrane Database Syst Rev 2018; 6: CD006650.

9. Lee AY, Levine MN, Baker RI et al. Low-molecular-weight heparin versus a coumarin for the prevention of recurrent venous thromboembolism in patients with cancer. N Engl J Med 2003; 349(2): $146-153$.

10. Lee AY, Kamphuisen PW, Meyer G et al. Tinzaparin vs Warfarin for Treatment of Acute Venous Thromboembolism in Patients With Active
Cancer: A Randomized Clinical Trial. JAMA 2015; 314(7): 677-686.

11. Kearon C, Akl EA, Ornelas J et al. Antithrombotic Therapy for VTE Disease: CHEST Guideline and Expert Panel Report. Chest 2016; 149(2): 315-352.

12. Farge $D$, Bounameaux $H$, Brenner $B$ et al.Inter national clinical practice guidelines including guidance for direct oral anticoagulants in the treatment and prophylaxis of venous thromboembolism in patients with cancer. Lancet Oncol 2016; 17(10): e452-e466.

13. Khorana AA, Yannicelli D, McCrae KR et al. Evaluation of US prescription patterns: Are treatment guidelines for cancer-associated venous thromboembolism being followed? Thromb Res 2016; 145: 51-53.

14. Khorana AA, McCrae KR, 15. Milentijevic D et al. 18. Current practice patterns and patient persistence with anticoagulant treatments for cancer】associated thrombosis. 20. Res Pract Thromb Haemost 2017; 1: 14-22.

15. Matzdorff A, Ledig B, Stuecker M, Riess H. Practice Patterns for Prophylaxis and Treatment of Venous Thromboembolism in German Cancer Patients. Oncol Res Treat 2016; 39(4): 194-201.

16. Bona RD, Sivjee KY, Hickey AD, Wallace DM, Wajcs SB. The efficacy and safety of oral anticoagulation in patients with cancer. Thromb Haemost 1995; 74(4): 1055-1058.

17. Marshall AL, Campigotto F, Neuberg D, Rowe B, Connors JM. Recurrence of Venous Thromboembolism in Patients With Cancer Treated With Warfarin. Clin Appl Thromb Hemost 2015; 21(7): 632-638.

18. Voigtlaender M, Langer F. Direct oral anticoagulants for the treatment of cancer-associated venous thromboembolism. What do we know so far? Hamostaseologie 2017; 37(4): 241-255.

19. Raskob GE, van Es N, Verhamme P, et al. Hokusai VTE Cancer Investigators. Edoxaban for the Treatment of Cancer-Associated Venous Thromboembolism. N Engl J Med 2018; 378(7): 615-624.

20. van Es N, Di Nisio M, Bleker SM, et al. Edoxaban for treatment of venous thromboembolism in patients with cancer. Rationale and design of the Hokusai VTE-cancer study. Thromb Haemost 2015; 114(6): 1268-1276

21. Young AM, Marshall A, Thirlwall J, et al. Comparison of an Oral Factor Xa Inhibitor With Low Molecular Weight Heparin in Patients With Cancer With Venous Thromboembolism: Results of a Randomized Trial (SELECT-D). J Clin Oncol 2018; 36(20): 2017-202322..

22. Li A, Garcia DA, Lyman GH, Carrier M. Direct oral anticoagulant (DOAC) versus low-molecularweight heparin (LMWH) for treatment of cancer associated thrombosis (CAT): A systematic review and meta-analysis. Thromb Res 2018 (Epub ahead of print)

23. Voigtlaender M, Langer F. A case-based practical approach to the management of cancer-associated venous thromboembolism. Vasa 2018; 47(2): $77-89$. 